\title{
Modelling and Control of Series HEVs Including Resistive Losses and Varying Engine Efficiency
}

\author{
Magnus Neuman ${ }^{1,2}$, Henrik Sandberg ${ }^{1}$, Bo Wahlberg $^{1}$ and Anders Folkesson ${ }^{2}$ \\ ${ }^{1}$ Automatic Control, School of Electrical Engineering, Royal Institute of Technology. \\ ${ }^{2}$ Scania CV AB.
}

Copyright $\odot 2008$ SAE International

\begin{abstract}
A model of a series HEV (Hybrid Electric Vehicle) including resistive losses in the energy storage and varying engine and generator efficiency is presented. The purpose is to study how system properties affect the optimal control of series HEVs. We show that there is an inherent trade-off in the control of such a system, based on the characteristics of the efficiency curve of the EGU (Engine and Generator Unit) together with the energy storage internal resistance, capacitance and energy level. The relation between these properties determines the optimal control of the EGU, and this is thus the mechanism behind all optimal control problems using a similar model setup. We illustrate our reasoning using deterministic dynamic programming where system properties are varied and the optimal control is studied.
\end{abstract}

\section{INTRODUCTION}

Hybrid Electric Vehicles (HEVs) are gaining importance as a means of reducing fuel consumption, emissions and noise. The key idea is to brake electrically thus reusing energy that otherwise would have been lost as heat. The electrical braking is done by an electric machine that is mechanically connected to the vehicle wheel axle. HEVs also allow for optimal operation of the combustion engine, since the electric machine can supply power during acceleration and cruising thereby altering the power demand of the combustion engine. That is, the electric machine works as propulsion motor. In a series HEV there is no mechanical connection between the propulsion motor and the combustion engine. Instead the combustion engine is directly coupled to a generator supplying the system with electrical power. In this way the combustion engine operation point is independent of vehicle velocity thus further increasing the possibilities of optimal operation. If there is a mechanical connection between the driven axle and the combustion engine it is termed a parallel HEV. A HEV is equipped with an energy storage such as supercapacitors or batteries that supplies or absorbs energy to or from the system. This work focuses on series HEVs with supercapacitors as energy storage.
Previous work on control of HEVs focus mainly on finding the optimal control. Methods in use include deterministic [1]-[3] and stochastic dynamic programming [4], [5], Pontryagin's minimum principle [6], [7] and game theory [8]. The optimal control generally depends on knowledge about the probability distribution of factors such as power load over a specific driving cycle, and transferring the optimal solution to a feasible real-time strategy has proved an awkward process. In a previous work we proposed a real-time strategy for a heavy series HEV based on the optimal control over standard city bus driving cycles [9]. Control of series HEVs has also been treated in [10]-[13], but none of them adopt the approach of this work.

In the present work we investigate how fundamental system properties such as energy storage internal resistance and capacity together with efficiency characteristics of the Engine and Generator Unit (EGU) affect the optimal control of series HEVs. Previous works generally use a setup of system parameters without studying how changing the parameters can affect their solution. Our purpose is thus to investigate the mechanism behind the optimal control of this kind of system and map how it is related to system properties. In this way we establish analytically the intuitive insight that the relation between energy storage and EGU efficiency determines the optimal control. This investigation also validates the model we present since it is tested if it can reproduce the behaviour that is intuitively expected.

\section{DISPOSITION OF THE PAPER}

In the next section a model of a series HEV is described. We then derive expressions to show how the relation between power losses in the energy storage and the EGU together with the power levels on the driving cycle affect the optimal control. Finally, deterministic dynamic programming is employed to illustrate our result. 


\section{MODELLING A SERIES HEV}

The series HEV is modelled using a power balance between the propulsion motor power $P_{p m}$, the generator power $P_{\text {gen }}$ and the supercapactitor power $P_{s c}$. This is expressed as

$$
0=P_{s c}(t)-P_{g e n}(t)+P_{p m}(t)
$$

where $P_{p m}<0$ when energy is input to the system.

While the driver behaviour determines $P_{p m}$ the quantity that can be controlled is $P_{\text {gen }}$, and the supercapacitors supply or absorb power according to (1). Fig. 1 shows a schematic illustration of the model setup. We neglect all effects of powertrain inertia in this analysis.

\section{ENERGY STORAGE MODEL}

The supercapacitors are modelled as a resistance connected in series with a capacitance. The change in energy content $E$ of the supercapacitors then becomes

$$
\begin{aligned}
\dot{E}(t) & =\frac{E(t)}{R C}\left(-1+\sqrt{1+\frac{2 R C P_{s c}(t)}{E(t)}}\right) \\
& =P_{s c}(t)-d_{e}\left(E(t), P_{s c}(t)\right)
\end{aligned}
$$

where we have defined $d_{e}\left(E(t), P_{s c}(t)\right)$ as the dissipation rate of the electrical system, adopting the notation of Willems in the classical paper on dissipative systems [14]. $R$ and $C$ are the supercapacitor resistance and capacitance. We thus have

$$
\begin{aligned}
& d_{e}\left(E(t), P_{s c}(t)\right)=P_{s c}(t)- \\
& \frac{E(t)}{R C}\left(-1+\sqrt{1+\frac{2 R C P_{s c}(t)}{E(t)}}\right)
\end{aligned}
$$

as an expression for the dissipated power.

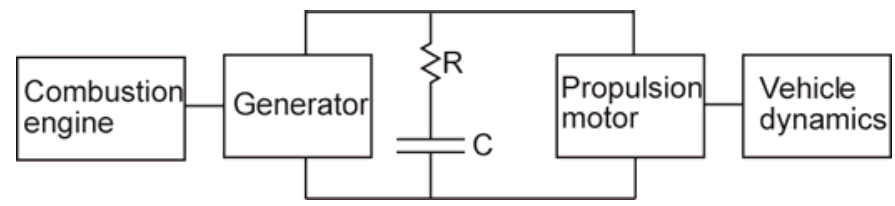

Figure 1: A schematic picture of the series HEV model. $R$ and $C$ are supercapacitor resistance and capacitance.

\section{ENGINE AND GENERATOR MODEL}

We use a simple static model of the EGU

$$
P_{\text {gen }}(t)=P_{\text {fuel }}(t)-d_{c}\left(P_{\text {fuel }}(t)\right)
$$

where $P_{\text {fuel }}$ denotes the power corresponding to the fuel used. The difference between input fuel power and the output generator power is the dissipation rate $d_{c}$ of the EGU. We assume it has the form

$$
\begin{aligned}
& d_{c}\left(P_{\text {fuel }}(t)\right)=P_{\text {fuel }}\left(1-\eta_{E G U}\right) \\
& =P_{\text {fuel }}\left[1-\eta_{1}\left(1-e^{-\beta_{1}\left(P_{\text {fuel }}-P_{\text {illing }}\right)}\right)-\eta_{2} e^{-\beta_{2}\left(P_{\text {fuel }}-P_{\text {fuel }}^{\prime}\right)^{2}}\right],
\end{aligned}
$$

where

$$
\eta_{E G U}=\eta_{1}\left(1-e^{-\beta_{1}\left(P_{\text {tuel }}-P_{\text {idling }}\right)}\right)+\eta_{2} e^{-\beta_{2}\left(P_{\text {fuel }}-P_{\text {tuel }}^{\prime}\right)^{2}}
$$

is the EGU efficiency. The parameters $\eta_{1}, \eta_{2}, \beta_{1}, \beta_{2}$ determine the shape of the efficiency curve, while $P_{\text {fuel }}^{\prime}$ positions the efficiency maximum of the EGU. Increasing $\beta_{2}$ gives a sharper efficiency peak centered around $P_{\text {fuel }}^{\prime}$. In the low power region the efficiency approaches zero since the EGU internal friction dominates. The rate at which the efficiency approaches zero is determined by $\beta_{1}$. The efficiency becomes zero at $P_{\text {idling }}$ which is the idling power loss. The parameters $\eta_{1}$ and $\eta_{2}$ determine the magnitude of the EGU efficiency.

This way of modelling the EGU captures the essentials of an EGU, that is, an optimal operation point and low efficiency at low powers, and it is possible to alter the characteristics of the EGU using the model. It compares reasonably to manufacturer data though the characteristics of the efficiency peak can differ, but still it serves the purpose of the present work. In Fig. 2 our model with realistic choices of parameters is shown together with typical values for the efficiency of a dieselEGU based on manufacturer data. We see that both the model and the actual EGU have an optimal operation point giving the maximum efficiency.

\section{MODEL ANALYSIS}

In this section we derive expressions for the instantaneous efficiency of the series hybrid powertrain. To improve readability, the time dependence of variables is not always stated explicitly.

\section{ENERGY STORAGE DISSIPATION}

In order to be able to do some analysis we simplify our model. We Taylor expand the dissipation rate of the energy storage as 


$$
d_{e}\left(E(t), P_{s c}(t)\right)=\frac{R C P_{s c}^{2}(t)}{2 E}+O\left(P_{s c}^{3} / E^{2}\right)
$$

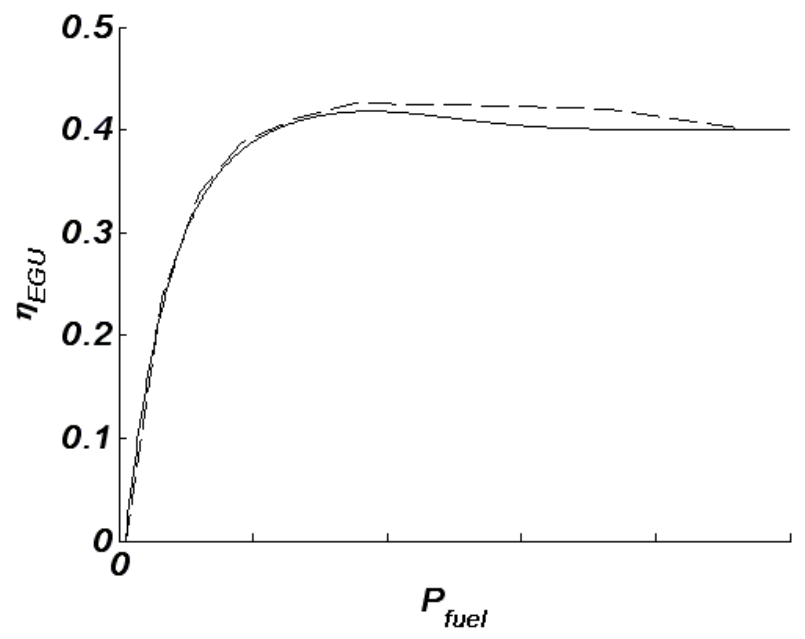

Figure 2: Efficiency of the engine and generator unit (EGU) as a function of input fuel power. The model presented here corresponds to the solid line while the dashed line is based on manufacturer data. In both cases there is an optimal operation point giving the maximum efficiency.

and we immediately see that the dissipation rate increases as $P_{s c}^{2}$ and that it decreases as $E / R C$ increases. Hence, the dissipation rate depends on the magnitude of the power (independent of sign), and is smaller when there are large amounts of energy in the capacitor relative to the magnitude of $R C$.

Note that it holds that

$$
\begin{array}{ll}
d_{e}\left(E_{1}, P_{s c}\right) \geq d_{e}\left(E_{2}, P_{s c}\right), & E_{2} \geq E_{1} \\
d_{e}\left(E, P_{s c, 1}\right) \geq d_{e}\left(E, P_{s c, 2}\right), & P_{s c, 1} \geq P_{s c, 2} \geq 0 \\
d_{e}\left(E, P_{s c, 1}\right) \geq d_{e}\left(E, P_{s c, 2}\right), & P_{s c, 1} \leq P_{s c, 2} \leq 0
\end{array}
$$

$\forall E, P_{s c}$. This means that more energy is dissipated at low energy levels and high power levels.

\section{DISCHARGING THE ENERGY STORAGE}

The instantaneous efficiency when discharging the energy storage is

$$
\eta_{s c, \text { disch. }}=\frac{-P_{s c}}{-P_{s c}+d_{e}} \approx \frac{2 E}{2 E-R C P_{s c}}
$$

using the expression in (7). Note that $P_{s c}<0$ during discharge. This also indicates that we should have high energy levels, low $R C$ and low $P_{s c}$.

\section{CHARGING THE ENERGY STORAGE}

During normal operation when charging, or not using, the energy storage the total instantaneous efficiency is given by

$$
\eta_{\text {total }}=\frac{P_{\text {fuel }}-P_{p m}-d_{c}\left(P_{\text {fuel }}\right)-d_{e}\left(P_{\text {gen }}-P_{p m}\right)}{P_{\text {fuel }}-P_{p m}}
$$

The objective is to maximize the total efficiency $\eta_{\text {total }}$. Since $P_{p m}$ is electrical power and we neglect the losses in the power electronics, the only effect of $P_{p m}$ is to alter the power level in the system. For the purpose of this analysis we can then put $P_{p m}=0$. We thus obtain

$$
\begin{aligned}
\eta_{\text {total }} & =\eta_{E G U}\left(P_{\text {fuel }}\right)-\frac{d_{e}\left(P_{\text {fuel }}-d_{c}\left(P_{\text {fuel }}\right)\right)}{P_{\text {fuel }}} \\
& \approx \eta_{E G U}\left(P_{\text {fuel }}\right)-\frac{R C\left(P_{\text {fuel }}-d_{c}\left(P_{\text {fuel }}\right)\right)^{2}}{2 E P_{\text {fuel }}} \\
& =\eta_{E G U}\left(P_{\text {fuel }}\right)-\frac{R C}{2 E} P_{\text {fuel }} \eta_{E G U}\left(P_{\text {fuel }}\right)^{2}
\end{aligned}
$$

as an expression for the total efficiency. In the second approximate equality (7) is used. Hence, the total efficiency $\eta_{\text {total }}$ deviates most from $\eta_{E G U}$ when the product $P_{\text {fuel }} \eta_{E G U}\left(P_{\text {fuel }}\right)^{2}$ is large. We see that there is an interesting trade-off between the terms in (11). The first term typically tends to increase $P_{\text {fuel }}$, whereas the second term typically tends to decrease $P_{\text {fuel }}$. Using some further approximations, we will analyze this behavior next.

Assume that it is optimal to run the EGU by itself at some power $P_{\text {fuel }}=P_{\text {fuel }}^{*}$. Since $P_{\text {fuel }}^{*}$ is assumed to be a local maximum of $\eta_{E G U}$ at $\eta_{E G U}^{*}$, in a neighborhood around $P_{\text {fuel }}^{*}$, we have

$$
\begin{aligned}
& \eta_{E G U}\left(P_{\text {fuel }}\right) \approx \eta_{E G U}^{*}-\beta \Delta P_{\text {fuel }}^{2}, \\
& \Delta P_{\text {fuel }}=P_{\text {fuel }}-P_{\text {fuel }}^{*}
\end{aligned}
$$

for some $\beta \geq 0$. Inserting (12) in (11) gives

$$
\begin{aligned}
\eta_{\text {total }} \approx & \eta_{E G U}^{*}\left(1-\eta_{E G U}^{*} R C P_{\text {fuel }}^{*} / 2 E\right) \\
& -\left(\eta_{E G U}^{*}\right)^{2}(R C / 2 E) \Delta P_{\text {fuel }} \\
& -\beta\left(1-\eta_{E G U}^{*} R C P_{\text {fuel }}^{*} / E\right) \Delta P_{\text {fuel }}^{2}
\end{aligned}
$$


where only up to quadratic terms in $\Delta P_{\text {fuel }}$ are kept. For realistic parameters $\beta\left(1-\eta_{E G U}^{*} R C P_{\text {fuel }}^{*} / E\right)>0$ holds, and there is a local maximum of (13) corresponding to

$$
P_{\text {fuel,new }}^{*}=P_{\text {fuel }}^{*}-\frac{\left(\eta_{E G U}^{*}\right)^{2}}{4 \beta\left(E / R C-\eta_{E G U}^{*} P_{\text {fuel }}^{*}\right)} .
$$

Equation (14) thus gives the new optimal operation point $P_{\text {fuel,new }}^{*}$ of the EGU when both the efficiency of the energy storage and the EGU are taken into account. We see that the deviation from $P_{\text {fuel }}^{*}$ depends on $\beta$, which captures the characteristics of the maximum of $\eta_{E G U}$, in a way similar to $\beta_{2}$ in (5). If $\beta$ is increased the efficiency peak becomes more distinct and it is optimal to stay at $P_{\text {fuel }}^{*}$, since the second term in (14) becomes small. Decreasing $\beta$ evens out the EGU efficiency curve, and it is optimal to run the EGU at other powers than $P_{\text {fuel }}^{*}$, since the second term in (14) becomes significant. At the same time, the fraction $E / R C$ influences the optimal operation point in the same way. That is, if $E / R C$ is large the optimal operation point is mainly determined by $P_{\text {fuel }}^{*}$, but if $E / R C$ decreases it is optimal to lower the EGU power. Including the time dependence, this fraction is $\frac{E(t) / C}{R}$. Hence, it is small if the energy level is normally low during operation and/or the internal resistance is high, and vice versa.

Naturally, (11) also tells us that when not using the energy storage the total efficiency is equal to the EGU efficiency.

DEPENDENCE OF THE OPTIMAL CONTROL ON VEHICLE PARAMETERS

To summarize the results so far, we have:

- Keep $R C$ low, $P_{\text {sc }}$ low and $E$ high to minimize losses in the energy storage.

- If $\beta$ is large, i.e., the EGU has a distinct efficiency peak, run the EGU in $P_{\text {fuel }}^{*}$ if $E(t) / R C$ is large enough.

- For small $\beta$ it can be optimal not to run the EGU in $P_{\text {fuel }}^{*}$ if $E(t) / R C$ is small enough.

These results express the trade-off inherent in the optimal control of this kind of systems. It is based on the relation between $\beta$ and $E / R C$, which expresses efficiency characteristics of the EGU and energy storage internal resistance, capacitance and energy level. Calculating the optimal control is simply determining what values of $\beta$ and $E / R C$ that should be considered. This trade-off is the mechanism behind all optimal control problems based on a model involving resistive losses and varying engine efficiency.

To clarify the consequences of these results we investigate what happens at extreme values of the parameters. A possible powertrain setup would be a small vehicle with large energy storage with relatively small internal resistance, i.e., $E / R C$ is typically large during operation. If the EGU has a distinct efficiency peak, $\beta$ is large. Equation (14) then tells us that the EGU should be operated in $P_{\text {fuel }}^{*}$. The optimal control strategy in this case is thus a thermostat or on-off strategy running the EGU in the optimal operation point. Since the vehicle is small, the power levels involved are low during operation. Equation (11) then tells us that the total efficiency $\eta_{\text {total }}$ is mainly determined by $\eta_{E G U}$, which also indicates that the EGU should be operated in $P_{\text {fuel }}^{*}$.

On the other extreme, we have a heavy vehicle with relatively small energy storage with high internal resistance together with small variations of EGU efficiency over a wide power range. That is, $E / R C$ and $\beta$ are small while the power levels are high. In this case it is less obvious what determines the total efficiency since the term $\left(\eta_{E G U}^{*}\right)^{2} /\left[4 \beta\left(E / R C-\eta_{E G U}^{*} P_{\text {fuel }}^{*}\right)\right] \quad$ becomes significant. However, we can conclude that $P_{\text {fuel }}$ must be carefully controlled since $P_{\text {fuel,new }}^{*}$ can change significantly during operation. Also, since the power levels are high, choosing the wrong $P_{\text {fuel }}$ has large consequences on the total efficiency. Since $E$ will vary significantly in this powertrain setup, it is wise to monitor $E$ and base the control on this measurement, in this way assuring that we operate the EGU as close to $P_{\text {fuel,new }}^{*}$ as possible. This also leads us to the conclusion that the control of heavy HEVs using small energy storages with high internal resistance is not as straightforward as the control of small vehicles using large energy storages with low internal resistance. In Fig. 3 this result is shown schematically.

So far only the instantaneous efficiency has been treated. If we consider a whole driving cycle, what is instantaneously optimal is not necessarily optimal globally. To investigate if our conclusions hold when we consider a time history we employ dynamic programming. 


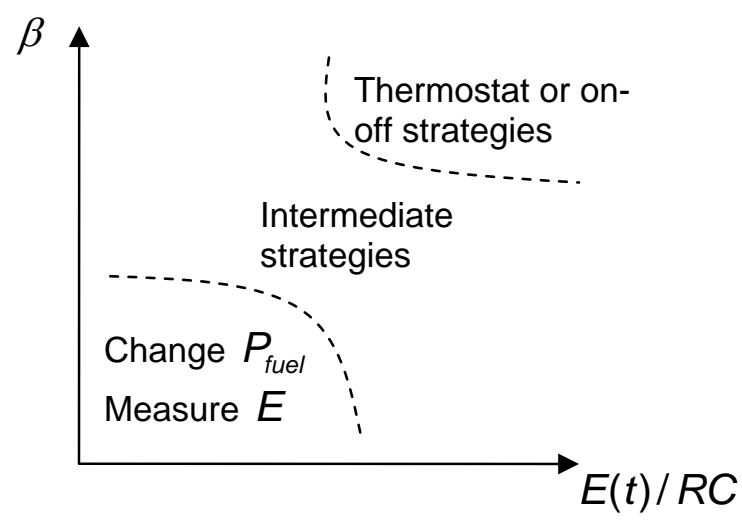

Figure 3: The dependence of the optimal control strategy on vehicle configuration, represented as $\beta$ and $E(t) / R C$. Large energy storages with high internal resistance together with a distinct efficiency peak of the EGU leads to on-off or thermostat strategies.

\section{DYNAMIC PROGRAMMING ANALYSIS}

To solve the optimal control problem it is discretisized on a time and state grid over the time interval $[0, N]$ with time step $\Delta t$. It then becomes

$$
\begin{aligned}
& \min \phi\left(E_{N}-E_{0}\right)+\sum_{k=0}^{N-1} P_{\text {fuel }, k} \\
& \text { subj. to }\left\{\begin{array}{l}
E_{k+1}=E_{k}+\left(P_{g e n, k}-P_{p m, k}-d_{e, k}\right) \Delta t \\
P_{f u e l, k}=P_{g e n, k}+d_{c, k} \\
E_{k} \in\left[E_{\text {min }}, E_{\text {max }}\right] \\
P_{g e n, k} \in S_{k} \cap\left[P_{g e n, \text { min }}, P_{\text {gen,max }}\right]
\end{array}\right\}
\end{aligned}
$$

where $k$ is the time index. We thus minimize $P_{\text {fuel }}$ and the relation between control signal $P_{\text {gen }}$ and $P_{\text {fuel }}$ is given by (4). The function $\phi\left(E_{N}-E_{0}\right)$ ensures that the final state $E_{N}$ is equal to the initial state $E_{0}$ by penalizing deviations. The set of admissible controls $S_{k}$ is time varying and contains the controls taking the system from the current state to states reachable in the next time step. If the time and state grid is made too coarse, the solution will contain discretization noise. It is therefore important to choose the resolution of the grid carefully.

We assume that the energy storage supplies the system with the necessary power, and we thus put no constraint on the maximum or minimum power of this component.

A problem of this type is conveniently solved using dynamic programming since it handles nonlinearities and constraints in a straightforward way [15]. Dynamic programming is based on the Bellman equation and solves the problem via backwards recursion:

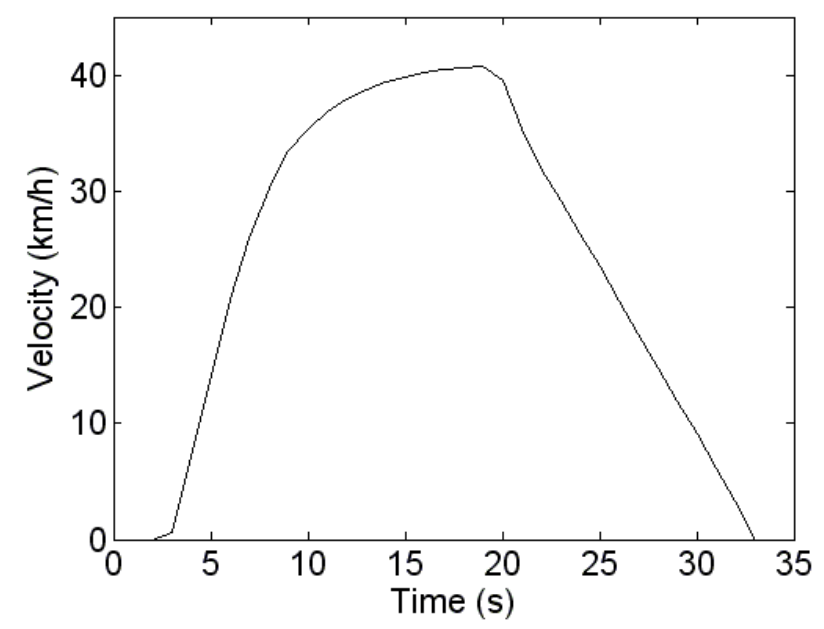

Figure 4: The velocity profile of the optimal control problem.

$$
J^{*}\left(n, E_{n}\right)=\min _{P_{\text {gen }, n}}\left\{P_{\text {fuel }, n}+J\left(n+1, E_{n+1}\right)\right\}
$$

where $J^{*}\left(n, E_{n}\right)$ is the optimal cost-to-go at time instant $n$ and state $E_{n}$.

We construct a driving cycle and obtain $P_{p m}$ necessary for solving (15) using a comprehensive simulation model developed in-house at Scania. It is essentially based on standard models of vehicle dynamics. To facilitate evaluation of the optimal control, we choose a simple velocity profile shown in Fig. 4. This consists of acceleration, constant speed and deceleration phases, and should give good insight into how system properties affect the optimal control.

We solve (15) on this driving cycle for two vehicle setups. To investigate if the conclusions from the previous section hold, the setups chosen differ in $R C$ and $\beta$. Their respective efficiency curves are shown in Fig. 5.

Setup 1 has a distinct efficiency peak and thus a high $\beta$-value, $\beta_{\text {high }}$. Setup 2 has a less distinct efficiency peak and $\beta=\beta_{\text {low }}$. To allow for EGU shut-off, $P_{\text {idling }}$ is put to zero, and there is thus no fuel injection when $P_{\text {gen }}$ is zero.

We use $R_{1} C_{1}=100 R_{2} C_{2}$ to vary the energy storage characteristics. The vehicle setups are thus:

$$
\text { 1. } \beta_{\text {high }}, R_{1} C_{1}
$$


2. $\beta_{\text {low }}, R_{2} C_{2}$

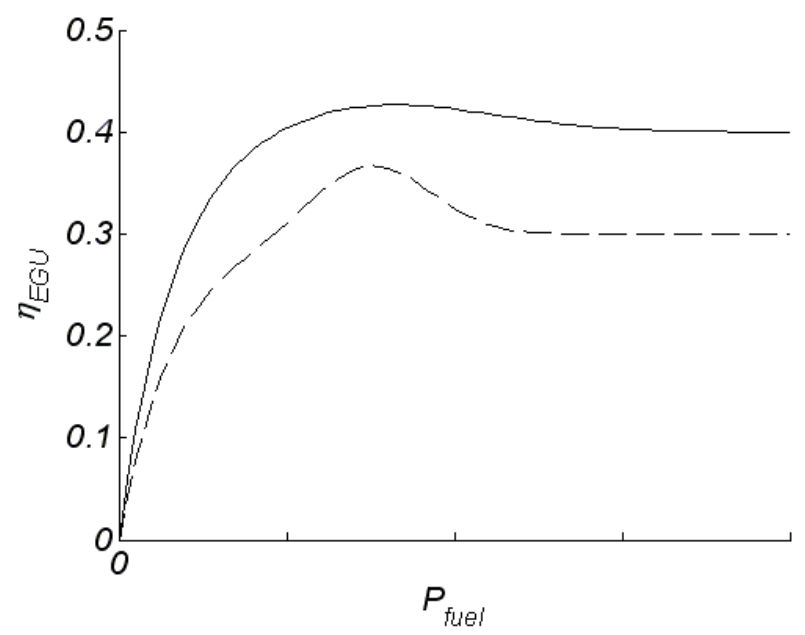

Figure 5: The engine and generator efficiency for the two cases studied using dynamic programming. Dashed curve has a high $\beta$-value and solid curve has a low.

with $R_{1} C_{1}>R_{2} C_{2}$.

Setup 1 can correspond to a vehicle using an otto engine and relatively large energy storage with low internal resistance. Setup 2 can be a diesel engine vehicle with small energy storage with high internal resistance.

Typically, $\quad \beta_{\text {diesel engine }}<\beta_{\text {fuel cell }}<\beta_{\text {otto engine }}$. Battery based energy storages have larger storage capacity but also higher internal resistance compared to a storage based on supercapacitors.

The optimal control $P_{\text {gen }}^{*}$ of the respective vehicle setups on the velocity profile in Fig. 4 is shown in Fig. 6. We see that Setup 1 has a characteristic on-off, or bangbang, behavior where the EGU is either operated at maximum efficiency or shut off. Having knowledge about the future time history of the system, the dynamic programming solution method chooses the optimal time to switch. This would not be possible in real-time and some criterion for when to switch would have to be defined. The optimal control of Setup 2 is not bangbang. Instead it follows the propulsion motor power in the acceleration up to a certain point, and then back to zero. This control ensures that the energy level is high, thus minimizing dissipation in the energy storage. These results are in accordance with the ones derived in the previous section. The trade-off when looking at the instantaneous efficiency thus also holds over a driving cycle.

\section{CONCLUSIONS}

A simple model of a series HEV was described. Its characteristic features are energy storage internal resistance and varying efficiency of the EGU. Using some simplifications, we were able to show analytically that the optimal operation point of the EGU depends on these characteristic features. The properties of the EGU

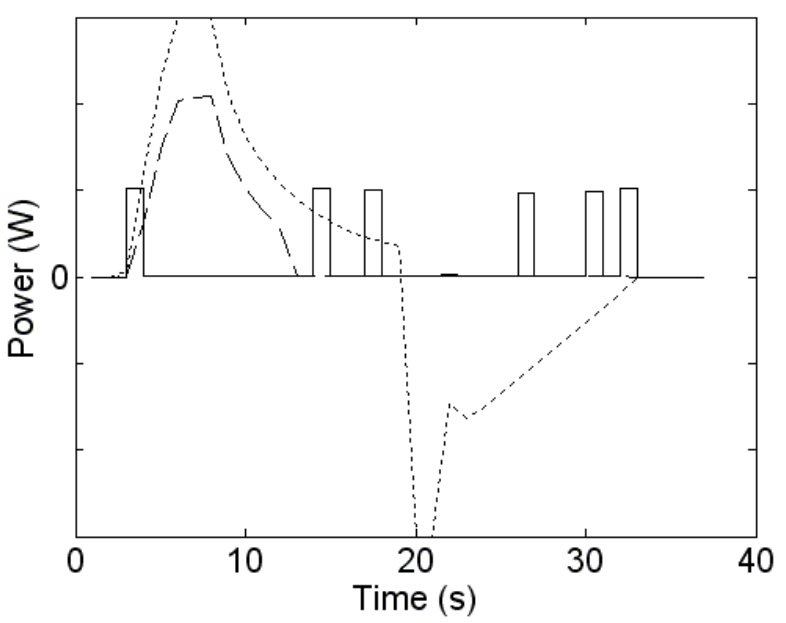

Figure 6: The optimal control $P_{\text {gen }}^{*}$ of the two vehicle setups over the chosen velocity profile. The propulsion motor power corresponds to the dotted line. The optimal control of Setup 1, with $\beta_{\text {high }}$ and $R_{1} C_{1}$, is shown as a solid line. The optimal control of Setup 2, with $\beta_{\text {low }}$ and $R_{2} C_{2}$, is the dashed line. We see that the first vehicle setup has a distinct on-off behavior, while Setup 2 is not on-off.

maximum efficiency operation point are here described by the sharpness of the EGU efficiency peak $\beta$, with a higher $\beta$ giving a more distinct efficiency peak. The optimal operation point of the EGU depends on $\beta$ and on the value of $E(t) / R C . E(t) / R C$ is large if the energy level is large during operation and the internal resistance is low, and vice versa. For large values of $\beta$ and $E(t) / R C$ the optimal operation point of the EGU is not altered by the inclusion of the energy storage, which should result in on-off strategies operating the EGU at maximum efficiency. If $\beta$ and $E(t) / R C$ are small, the optimal operation point is significantly altered by the inclusion of the energy storage, and should most likely change during operation. The analysis indicates that we should measure $E(t)$ to determine the optimal operation point. Our conclusions based on instantaneous efficiency were tested on a complete driving cycle using deterministic dynamic programming. The optimal control turned out to correspond to what intuition told us based on the analytical results. 
The main conclusion is thus that the optimal control of series HEVs can differ significantly depending on powertrain characteristics. An EGU with a distinct efficiency peak together with an energy storage with low internal resistance and high energy levels during operation results in on-off strategies. On the other hand, an EGU with a less distinct efficiency peak together with an energy storage with high internal resistance which is operated at lower energy levels results in a completely different control, which is non-trivial to determine. This conclusion is somewhat intuitive, but a contribution of the present work is to investigate this issue analytically. Intermediate cases should exhibit a mix of the strategies, and the strategy could also possibly vary during operation. This makes the optimal control of this kind of systems still more difficult. The trade-off between these powertrain characteristics is the mechanism behind all optimal control problems using a similar model setup.

Besides the powertrain characteristics investigated here there can be many other factors that affect the optimal control. If the cost function is modified to include for example noise, drivability, passenger comfort, engine transients or emissions, the optimal control can be different. This could possibly decrease the feasibility of an on-off strategy. In this work we focused on supercapacitors, but other types of energy storages are also interesting for optimal control problems. Though, modelling for example batteries is likely to become more complicated than supercapacitors.

\section{ACKNOWLEDGMENTS}

This work was sponsored by Scania $C V A B$ and Vinnova.

\section{REFERENCES}

1. LIN, C-C., PENG, H. and GRIZZLE, J.W., "Power Management Strategy for a Parallel Hybrid Electric Truck", IEEE Transactions on Control Systems Technology, vol. 11, 2003, pp. 839-849.

2. MUSARDO, C., RIZZONI, G. and STACCIA, B., "AECMS: An Adaptive Algorithm for Hybrid Electric Vehicle Energy Management", Proceedings of the 44th Conference on Decision and Control, and the European Control Conference, Seville, Spain, 2005, pp. 1816-1823.

3. ZHU, Y., CHEN, Y., TIAN, G., WU, H., and CHEN, Q., "A Four-Step Method to Design an Energy Management Strategy for Hybrid Vehicles", Proceedings of the 2004 American Control Conference, Boston, MA, 2004, pp. 156-161.

4. LIN, C-C., PENG, H. and GRIZZLE, J.W., "A Stochastic Control Strategy for Hybrid Electric Vehicles", Proceedings of the 2004 American
Control Conference, Boston, MA, 2004, pp. 47104715.

5. JOHANNESSON, L., ÅSBOGÅRD, M., and EGARDT, B., "Assessing the Potential of Predictive Control for Hybrid Vehicle Powertrains Using Stochastic Dynamic Programming", IEEE Transactions on Intelligent Transportation Systems, vol. 8, 2007, pp. 71-83.

6. DELPRAT, S., LAUBER, J., GUERRA, T. M., and RIMAUX, J., Control of a Parallel Hybrid Powertrain: Optimal Control, IEEE Transactions on Vehicular Technology, vol. 53, 2007, pp. 872-881.

7. SERRAO, L., and RIZZONI, G., "Optimal Control of Power Split for a Hybrid Electric Refuse Vehicle", 2008 American Control Conference, Seattle, WA, 2008, pp. 4498-4503.

8. DEXTREIT, C., ASSADIAN, F., KOLMANOVSKY, I. V., MAHTANI, J. and BURNHAM, K., "Hybrid Electric Vehicle Energy Management Using Game Theory", SAE World Congress, Detroit, MI, SAEPaper 2008-01-1317, 2008.

9. NEUMAN, M., SANDBERG, $\mathrm{H}$. and WAHLBERG, B., "Rule-Based Control of Series HEVs Derived from Deterministic Dynamic Programming", Submitted to American Control Conference 2009.

10. KONEV, A., LEZHNEV, L. and KOLMANOVSKY, I., "Control Strategy Optimization for a Series Hybrid Vehicle", SAE World Congress, Detroit, MI, SAEPaper 2006-01-0663, 2006.

11. JALIL, N., KHEIR, N. A. and SALMAN, M., "A RuleBased Energy Management Strategy for a Series Hybrid Vehicle", Proceedings of the American Control Conference, Albuquerque, NM, 1997, pp. 689-693.

12. BARSALI, S., MIULLI, C., and POSSENTI, A., "A Control Strategy to Minimize Fuel Consumption of Series Hybrid Electric Vehicles", IEEE Transactions on Energy Conversion, vol. 19, 2004, pp. 187-195.

13. BRAHMA, A., GUEZENNEC, Y., and RIZZONI, G., "Optimal Energy Management in Series Hybrid Electric Vehicles", Proceedings of the American Control Conference, Chicago, IL, 2000, pp. 60-64.

14. WILLEMS, J.C., "Dissipative Dynamical Systems, Part I: General theory; Part II: Linear Systems with Quadratic Supply Rates", Archive for Rationale Mechanics Analysis, vol. 45, 1972, pp. 321-393.

15. BERTSEKAS, D., Dynamic Programming and Optimal Control, Athena Scientific, Belmont, MA; 2007.

\section{CONTACT}

Corresponding author: Magnus Neuman, Scania CV AB, SE-151 87, Södertälje, Sweden. E-mail: magnus.neuman@scania.com, magnus.neuman@ee.kth.se. 